\title{
CFP and YFP, but Not GFP, Provide Stable Fluorescent Marking of Rat Hepatic Adult Stem Cells
}

\author{
Rouzbeh R. Taghizadeh and James L. Sherley \\ Programs in Regenerative Biology and Cancer, Boston Biomedical Research Institute, Watertown, MA 02472, USA \\ Correspondence should be addressed to James L. Sherley, sherleyj@bbri.org
}

Received 27 November 2007; Revised 10 January 2008; Accepted 2 February 2008

Recommended by Mouldy Sioud

The stable expression of reporter genes in adult stem cells (ASCs) has important applications in stem cell biology. The ability to integrate a noncytotoxic, fluorescent reporter gene into the genome of ASCs with the capability to track ASCs and their progeny is particularly desirable for transplantation studies. The use of fluorescent proteins has greatly aided the investigations of protein and cell function on short-time scales. In contrast, the obtainment of stably expressing cell strains with low variability in expression for studies on longer-time scales is often problematic. We show that this difficulty is partly due to the cytotoxicity of a commonly used reporter, green fluorescent protein (GFP). To avoid GFP-specific toxicity effects during attempts to stably mark a rat hepatic ASC strain and, therefore, obtain stable, long-term fluorescent ASCs, we evaluated cyan fluorescent protein (CFP) and yellow fluorescent protein (YFP), in addition to GFP. Although we were unable to derive stable GFP-expressing strains, stable fluorescent clones (up to 140 doublings) expressing either CFP or YFP were established. When fluorescently marked ASCs were induced to produce differentiated progeny cells, stable fluorescence expression was maintained. This property is essential for studies that track fluorescently marked ASCs and their differentiated progeny in transplantation studies.

Copyright ( 92008 R. R. Taghizadeh and J. L. Sherley. This is an open access article distributed under the Creative Commons Attribution License, which permits unrestricted use, distribution, and reproduction in any medium, provided the original work is properly cited.

\section{INTRODUCTION}

Fluorescent proteins have become widely used as markers for positively identifying and tracking expressing cells in many in vitro and in vivo studies. The most widely used, green fluorescent protein (GFP), cloned from Aequorea victoria, does not require substrates or cofactors and can be used in a variety of species $[1,2]$. Among its various uses as a marker, GFP has been used for transient studies of cell apoptosis [3], cytoskeletal dynamics [4], and inhibitory gene expression [5]. Since no cofactors are needed for the native GFP protein to develop fluorescence, it has been possible to use it as a marker in many different organisms. For instance, transgenic Drosophila melanogaster [6], zebrafish [7, 8], mice [9$12]$, and rats $[13,14]$ have been successfully derived using wild-type GFP. Although successful in obtaining stable GFPexpressing transgenic animals, attempts to develop in vitrocell lines stably expressing GFP have been largely unsuccessful $[2,3,15-18]$.

Currently, in adult stem cell (ASC) research, there is a critical need for methods to verify ASCs in vitro and track their progeny in in vivo repopulation studies. Since markers that uniquely identify ASCs are unknown, the accepted method to confirm the "stemness" of a cell population is by transplantation of cells into animals after injury to targeted tissues. If ASCs are present at significant levels in the transplanted cell population, the animal survives with repair of the damaged tissue. However, in these experiments, there is uncertainty as to whether the tissue has been repaired by the transplanted cells, by activated resident host ASCs, or by host cells recruited from another tissue source altogether. To overcome these uncertainties, methods to identify the transplanted cells and their descendents have been applied. Ideally, these methods need to identify donor cell progeny independent of subsequent differentiation status.

Though attractive as an in vivo tracking tool in ASC repopulation assays, GFP has several drawbacks. One shortcoming of GFP is that it can induce cell death. Intense excitation of the protein in vitro for extended periods can generate free radicals that are quite toxic to cells [15]. The inability to obtain constitutively expressing GFP cell strains may also be related to DNA methylation effects. In the presence 
of an irreversible inhibitor of methyl transferase, C3A human hepatoblastoma cells transfected with GFP showed a significantly greater retention of GFP expression and exhibited higher levels of GFP production [19]. As a result, GFP has been more successfully used extensively as a viable marker for mostly short-time scale experiments (hours), whereas attempts to establish long-term (months) GFP-expressing cell strains have been mostly unsuccessful [2, 3, 15-18]. The reported efficiency of establishing stable, constitutively expressing cell lines is extremely low [18].

GFP-expressing transgenic mice are readily available [912] and are a possible source for GFP-labeled cells. These mice are uniformly green with the exceptions of hair and red blood cells. However, there are still barriers to their use as a source of fluorescently marked ASCs. One major drawback is that, for many tissues, methods do not exist for efficient isolation of ASCs. Additionally, GFP-transgenic mice do not offer a solution for tracking ASCs derived from other species for which transgenic technology has not been developed.

Due to similar difficulties in developing ASCs with constitutive GFP expression, we evaluated two variants of GFP, cyan fluorescent protein (CFP) and yellow fluorescent protein (YFP) that have different excitation-emission profiles and, therefore, may have less toxicity associated with their free-radical byproducts. An early screen of $A$. victoria mutants produced CFP which has an emission spectrum below that of GFP due to a Tyr ${ }^{66}$ to $\operatorname{Trp}^{66}$ substitution [20,21]. Similarly, YFP has been rationally designed on the basis of the GFP crystal structure to red-shift the absorbance and emission spectra with respect to GFP. Based on these differences, we evaluated whether CFP and YFP might allow for stable, long-term fluorescence in rat hepatic ASCs derived in our laboratory. We were able to establish stable, long-term expressing ASC strains. When these fluorescently marked ASCs were induced to produce differentiated progeny cells, stable expression of fluorescence was maintained. Our findings indicate that CFP and YFP are more suitable markers for ASC studies in vitro and predict that they will be better markers for in vivo studies, as well.

\section{MATERIALS AND METHODS}

\subsection{Cells}

Previously derived [22] rat hepatic ASC strain, Lig-8, was maintained in Dulbecco's Modified Eagle Medium (DMEM; Life Technologies, Carlsbad, Calif, USA) supplemented with 10\% dialyzed fetal bovine serum (DFBS, JRH Biosciences, Lenexa, Kan, USA), 1\% Penicillin/Streptomycin (Life Technologies, Carlsbad, Calif, USA), and $400 \mu \mathrm{M}$ xanthosine (Xs; Sigma-Aldrich, St. Louis, Miss, USA) in a $37^{\circ} \mathrm{C}$ humidified incubator with a $5 \% \mathrm{CO}_{2}$ atmosphere.

\subsection{Plasmids}

Amplified plasmids for transfection were isolated by Qiagen (Valencia, Calif, USA) column fractionation as specified by the supplier and further purified by cesium chloride equilibrium density gradient centrifugation. Transfections were per- formed using $5 \mu \mathrm{g}$ of pEGFP-N3 vector plasmid (Clontech Laboratories, Palo Alto, Calif, USA) under the control of a cytomegalovirus (CMV) promoter. In addition, the pEGFP$\mathrm{N} 3$ vector contains a neomycin resistance gene insert under the control of the simian virus-40 (SV40) promoter conferring resistance to the antibiotic Genetecin ${ }^{\mathrm{TM}}$ (Life Technologies, Carlsbad, Calif, USA). The CFP and YFP derivatives of pEGFP-N3 were prepared by digestion and complete removal of the EGFP insert using BamH1 and Not1 endonucleases (New England Biolabs, Beverly, Mass, USA). The respective CFP or YFP insert was ligated into the vector after gel purification. Additional vectors used to attempt to derive stable GFP expressing cells included pCX-EGFP (supplied by B. Engleward, Massachusetts Institute of Technology) and pEGFP-N1 (Clontech Laboratories, Palo Alto, Calif, USA). pCX-EGFP regulates EGFP (enhanced GFP) under a chicken beta-actin promoter/CMV-IE enhancer and pEGFP-N1 is a sequence variant of pEGFP-N3.

\subsection{Transfection}

Lig- 8 cells were seeded at $1 / 10$ confluency in a $75-\mathrm{cm}^{2}$ flask (Corning, Corning, NY, USA) one day prior to transfection. Lig-8 cells were then independently transfected with the CFP, YFP, or GFP expression plasmids using Cytofectene (BioRad Laboratories, Hercules, Calif, USA), per manufacturer's suggested instructions. Approximately, $1.5 \times 10^{6}$ cells ( $1 / 5$ confluency in $75-\mathrm{cm}^{2}$ flask) were transfected for 16-20 hours and then given two phosphate buffer saline (PBS) washes, followed by supplementation with regular growth medium. The transfected cells were cultured for an additional 42-48 hours. After this time period, the transfected cells were replated at $1 / 6$ density in parallel in $10-\mathrm{cm}$ diameter plates (Corning, Corning, NY, USA). After overnight culture, the culture medium was replaced with medium supplemented to $0.5 \mathrm{mg} / \mathrm{ml}$ Genetecin ${ }^{\mathrm{TM}}$ to select for stably transfected cell clones.

\subsection{Clonal cell viability}

Propidium iodide (PI; Sigma, St. Louis, Mo, USA) was added directly to media and cells at $5 \mu \mathrm{g} / \mathrm{ml}$ to determine viability of clonal cells. A Nikon super high-pressure mercury lamp was used to image PI cells using a Nikon epifluorescent microscope.

\subsection{Derivation of fluorescent protein-expressing clones}

After 14 days in culture, transfected cells from each $10-\mathrm{cm}$ diameter plate were trypsinized and each transferred into a $75-\mathrm{cm}^{2}$ flask with selection medium maintained thereafter. After two days, the transfected cells were reseeded at 1000 cells each into five individual $10-\mathrm{cm}$ diameter dishes. Resistant colony formation was assessed after 10-14 days of culture in selection medium, with medium changes every 3 days. After this time, colonies were counted and scored as nonexpressing (B1; 0\% of cells in the colony were expressing fluorescent protein), semiexpressing (B2; estimated 25-75\% of cells in the colony were expressing fluorescent protein) 
TABLE 1: Relative transfection efficiency of fluorescent gene markers and cloning efficiency of selected transfected cell colonies. Transfection efficiency is defined as the average number of colonies/estimated number of cells transfected/ $\mu \mathrm{g}$ DNA. Transfections included $\sim 1.5 \times 10^{6}$ cells. Cloning efficiency is defined as the number of stable cell strains derived/number of colonies cultured.

\begin{tabular}{lccccc}
\hline $\begin{array}{l}\text { Fluorescent } \\
\text { marker }\end{array}$ & $\begin{array}{l}\text { Average colony } \\
\text { number/10-cm } \\
\text { dish }\end{array}$ & $\begin{array}{l}\text { Relative transfection } \\
\text { efficiency }\end{array}$ & $\begin{array}{l}\text { Number of } \\
\text { clones picked }\end{array}$ & $\begin{array}{l}\text { Number of Stable } \\
\text { cell strains derived }\end{array}$ & $\begin{array}{c}\text { Cloning efficiency } \\
\text { GFP }\end{array}$ \\
CFP & $1.3(n=10)$ & 0.02 & 8 & 0 & $0 \%$ \\
YFP & $64(n=3)$ & 1.00 & 6 & 6 & $100 \%$ \\
\hline
\end{tabular}

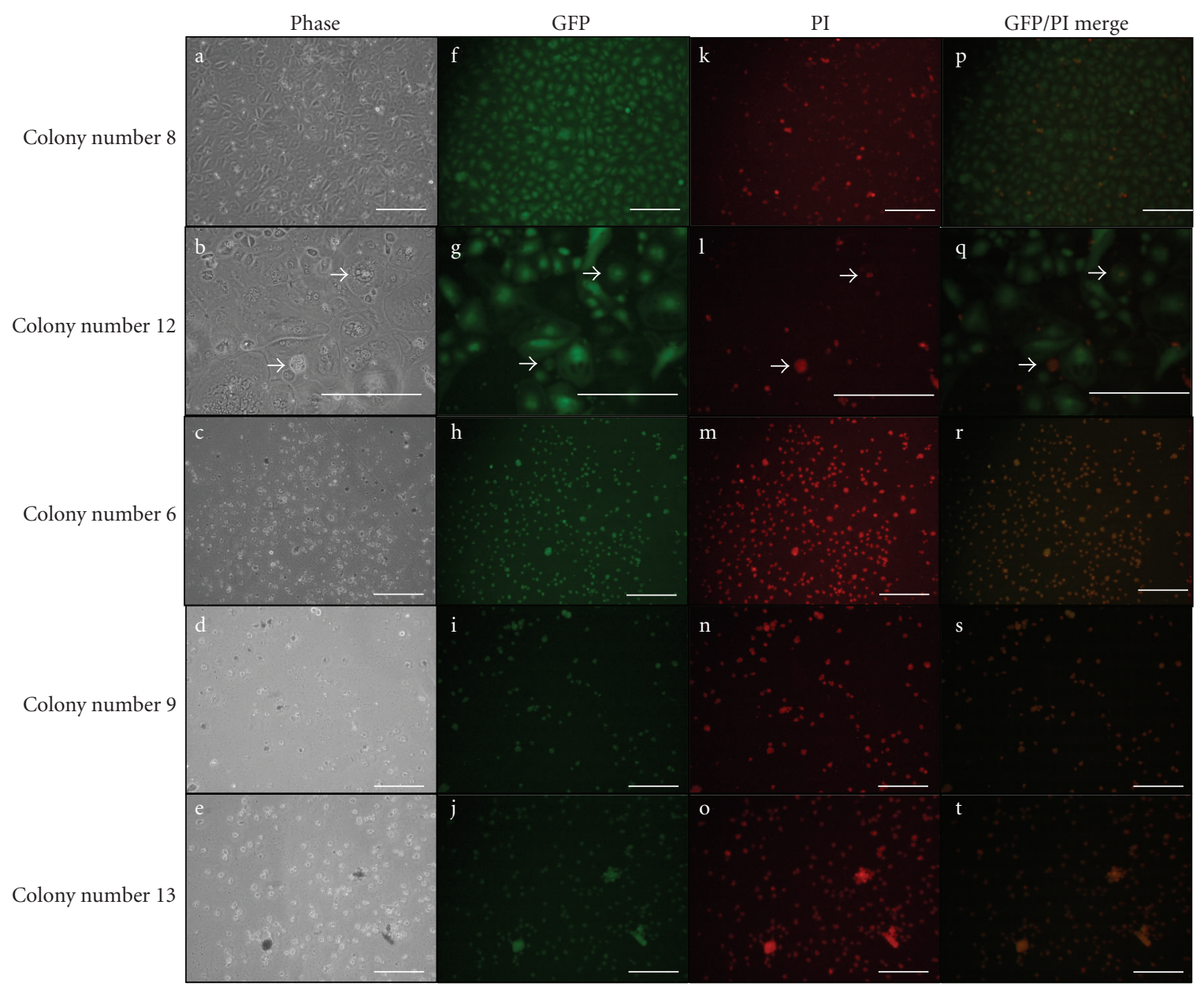

FIGURE 1: Transient, short-term GFP fluorescence expression by the rat hepatic ASC strain, Lig-8, is associated with cell death. Cell strain, Lig-8, was transfected with the pEGFP-N3 expression vector. Shown are images of 5 independent colonies transiently expressing GFP fluorescence at 72-96 hours posttransfection. Shown are the phases (a)-(e), GFP-fluorescence (f)-(j), propidium iodide (PI) fluorescence (k)-(o), and merged GFP and PI fluorescence (p)-(t) images. Arrows highlight specific examples of cells double-positive for GFP and PI fluorescence in Colony no. 12. Scale bar is equivalent to $100 \mu \mathrm{m}$.

or fullyexpressing (B3; $100 \%$ of the cells in the colony were expressing fluorescent protein). Several well-separated colonies were isolated within cloning cylinders (Bellco Glass, Vineland, NJ, USA), harvested by trypsinization, transferred to $25-\mathrm{cm}^{2}$ flasks (Corning, Corning, NY, USA), and allowed to expand for 10-14 days, until the flask was $>90 \%$ confluent. At that point, cells from the $25-\mathrm{cm}^{2}$ flasks were trypsinized and transferred into a $75-\mathrm{cm}^{2}$ flask. Within 3-4 days, the flasks were $90 \%$ confluent. After trypsinization, $\sim 80 \%$ of the cells in cultures of expanded clones were frozen in liquid nitrogen [22] for early passage stocks. All expanded clones exhibited respective CFP or YFP fluorescence expression for at least 15 population doublings before being cryogenically stored. The remaining cells were maintained in culture and 
passaged for at least 24 weeks (estimated 140 population doublings). Three of the CFP-expressing Lig- 8 clonal strains, $\mathrm{B} 1, \mathrm{~B} 2$, and B3, were subsequently evaluated for this study. Population doublings were determined based on the estimated total number of cells produced over time with the exponential generation time of $\sim 18$ hours determined for Lig- 8 parent cells.

\subsection{Flow cytometry and fluorescence microscopy}

A FACStar Plus flow cytometer (Becton, Dickinson and Company, Franklin Lakes, NJ, USA) was used to quantify the fraction of fluorescent cells in populations. The FACStar Plus was equipped with two coherent Innova 90 lasers for visible and ultraviolet argon lines. Data acquisition and analyses were performed with CellQuest software (Becton, Dickinson and Company, Franklin Lakes, NJ, USA) and Summit analyses software (Cytomation, Inc., Fort Collins, Colo), respectively. The nontransfected parent Lig-8 ASC strain was used as a negative control for all analyses to account for background cell autofluorescence. Cell populations were analyzed using both flow cytometry and epifluorescence microscopy using a Nikon TE300 microscope system with DAPI/GFP/CFP/YFP filters. A Hamamatsu digital camera and OpenLab imaging system were used for digital imaging. The Student's t test was used to determine the statistical confidence of observed differences in fluorescence.

\subsection{Differentiation induction}

Cells were treated for 9 days with $20 \mathrm{ng} / \mathrm{ml}$ epidermal growth factor (EGF) and $0.5 \mathrm{ng} / \mathrm{ml}$ transforming growth factor $\mathrm{b}$ (TGF-b) (Life Technologies, Carlsbad, Calif, USA), in the same culture medium, except that the DFBS was reduced to $1 \%$. The details of the differentiation induction protocol will be reported elsewhere [23].

\section{RESULTS}

Using CFP, YFP, or GFP as independent fluorescent protein markers, we transfected respective expression plasmids into a previously described rat hepatic ASC strain, Lig-8 [22]. Asymmetric selfrenewal associated with production of differentiated progeny cells is the defining feature of ASCs $[22,24]$. Lig-8 cells were derived based on their asymmetric cell kinetics $[22,24]$. Lig-8 cells asymmetrically self-renew and produce differentiated progeny with mature hepatocyte properties $[22,23,25]$. The differentiated progeny cells express transcription factors and protein antigens that are specific for hepatocyte development and maturation, respectively. Hepatocyte-specific phenotypes include binucleation, albumin secretion, and expression of inducible cytochrome P450s [22, 23, 25]. Based on well-defined ASC properties, Lig-8 cells were ideal for evaluation of effects of GFP, CFP, and YFP in ASCs.

We found that transfections with GFP gene constructs yielded transfected colonies $\sim 50$-fold less efficiently than transfections with the analogous CFP- or YFP-gene constructs (Table 1). In addition, colonies of GFP-transfected

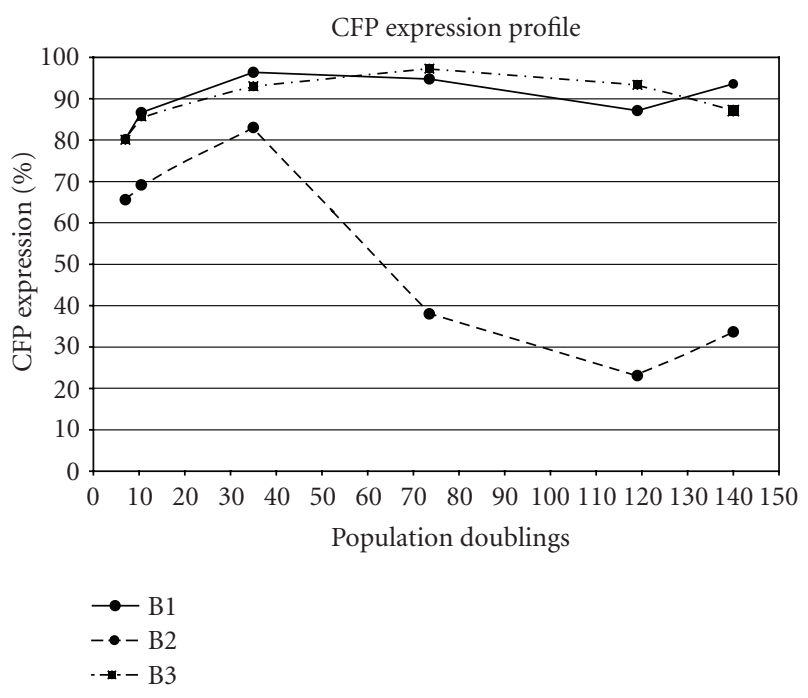

FIgURE 2: Stable, long-term expression of CFP fluorescence in stably transfected rat hepatic ASC clones. Three cell clones expanded from CFP-transfected colonies, B1, B2, and B3 (as described in text), were propagated and serially analyzed for CFP expression. At the indicated number of population doublings, the percentage of fluorescence-expressing cells was determined by flow cytometry for each specified clone. The cells have been passaged for a maximum of 140 population doublings.

cells could not be propagated as stable cell strains, whereas both CFP- and YFP-transfected cell colonies had 100\% cloning efficiency (Table 1). Furthermore, we determined that cells that were transiently-expressing GFP appeared to undergo cell death, as GFP-expressing cells were also positive for propidium iodide (PI) (Figure 1). PI is impermeable to intact membranes but readily penetrates the membranes of nonviable cells and binds to DNA and RNA, causing red fluorescence. The cells eventually rounded up and detached from the culture dish, while still showing GFP and PI fluorescence (Figure 1, colonies 6, 9, and 13). Similar observations were made with Lig- 8 cells transfected with pCX-EGFP and pEGFP-N1 plasmids. All observed GFP-expressing colonies yielded this same fate (data not shown).

Expanded CFP and YFP clones expressed the respective fluorescent proteins stably for at least 24 weeks in culture $(\sim 140$ doublings; e.g., clone B3 in Figure 2$)$. Clones that were successfully derived exhibited a range of CFP- or YFPexpressing cell fractions. As a result, these clones were characterized as nonexpressing (B1; $0 \%$ of cells in the colony express fluorescent protein, data not shown), semiexpressing (Figure 3, B2; at least 25-75\% of cells in the colony expressed fluorescent protein, but not all); or fully expressing (Figure 3, B3; approximately $100 \%$ of the cells in the colony expressed fluorescent protein). Although cell strains were derived from both CFP and YFP expressing colonies, only CFP cell strains were further evaluated.

Although, as colonies, the CFP-expressing cell strains exhibited the fluorescent properties described, with further propagation in culture, the fluorescence for one of the transfected clones decreased (B2 in Figure 2). Moreover, cells 


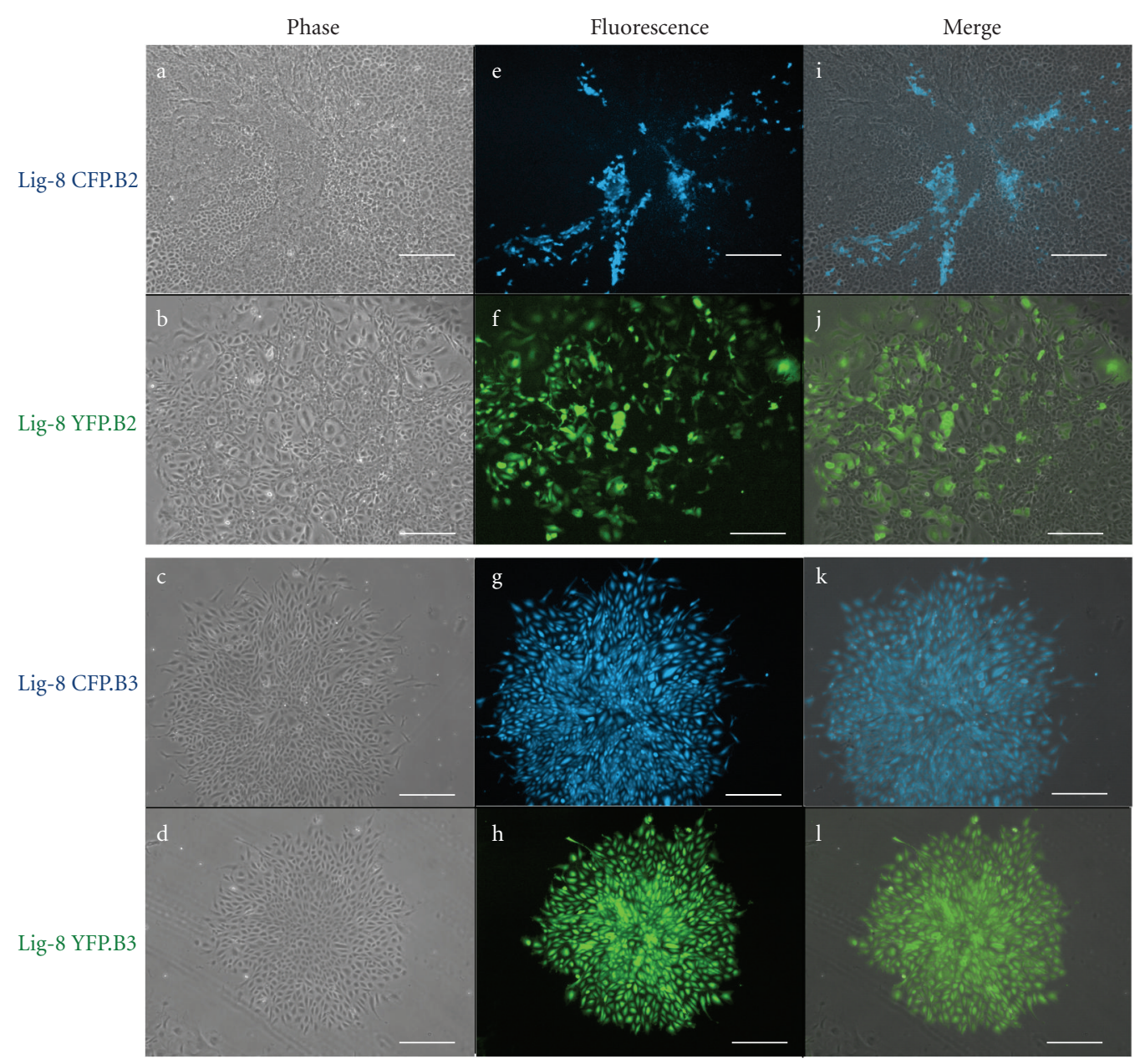

FIGURE 3: Fluorescent protein (CFP or YFP)-expressing colonies from rat hepatic ASCs. Rat hepatic ASC strain, Lig-8, was stably transfected with either a CFP or YFP expression vector. Shown are colonies with approximately $20-75 \%$ of the cells expressing (B2) and colonies with essentially $100 \%$ of the cells expressing (B3). Shown are the phases (a)-(d), fluorescent (e)-(h), and merged (i)-(l) images of CFP- and YFP-expressing colonies. Scale bar is equivalent to $100 \mu \mathrm{m}$.

derived from an initially non-expressing B1 colony began to express CFP (Figure 2; B1) at levels comparable to continuously, fully-expressing B3 clones (Figure 2; B3) during clonal propagation. Qualitatively, the B2 and B3 CFP-expressing clones maintained their initially observed fluorescent properties. Although there was some variation in expression seen in the early stages of clonal analysis, expression stabilized with propagation, and low variability was observed for at least 24 weeks (estimated 140 population doublings). The B2 cell clone exhibited the greatest fluctuation in fluorescence expression (Figure 2), but did not change in its basic character of expressing CFP.

Stable fluorescent protein expression did not alter the essential properties of the parent hepatic ASC strain, Lig-8 (data not shown). We have found that, because of their asymmetric self-renewal property, the parent Lig- 8 hepatic ASCs are resistant to complete differentiation by TGF- $\beta$, EGF, and serum deprivation [23]. Under conditions of TGF-b supplementation, Lig- 8 cells produce differentiated progeny cells by asymmetric cell divisions that allowed them to retain their undifferentiated ASC state [23]. To evaluate CFP expression in differentiated progeny cells, the three CFP-expressing fluorescent ASC strains (B1, B2, and B3) were examined after culture under EGF/TGF- $\beta$-induced differentiation conditions. All strains exhibited similar morphological and cell kinetic properties observed for the nontransfected parental Lig-8 strain (data not shown). As shown in Figure 4, under normal conditions, the B3 cell clone exhibited uniform cell morphology (a)-(c), whereas under differentiation conditions, a heterogeneous collection of varying morphological cell types appeared. Some differentiated cells had a noticeable larger size and altered morphology (Figure 4, (d)-(f); arrows), compared to cells under normal culture conditions.

After induction of differentiated progeny, the CFPfluorescent cell fraction of B1 cells did not vary significantly relative to the routine (undifferentiated) culture conditions (Table 2). However, although exhibiting stable fluorescence expression under differentiating conditions, B1 and B3 cell clones displayed a statistically significant increase $(60 \%, P<$ .01 ; and $91 \%, P<.002$, resp.) in median fluorescence per cell 

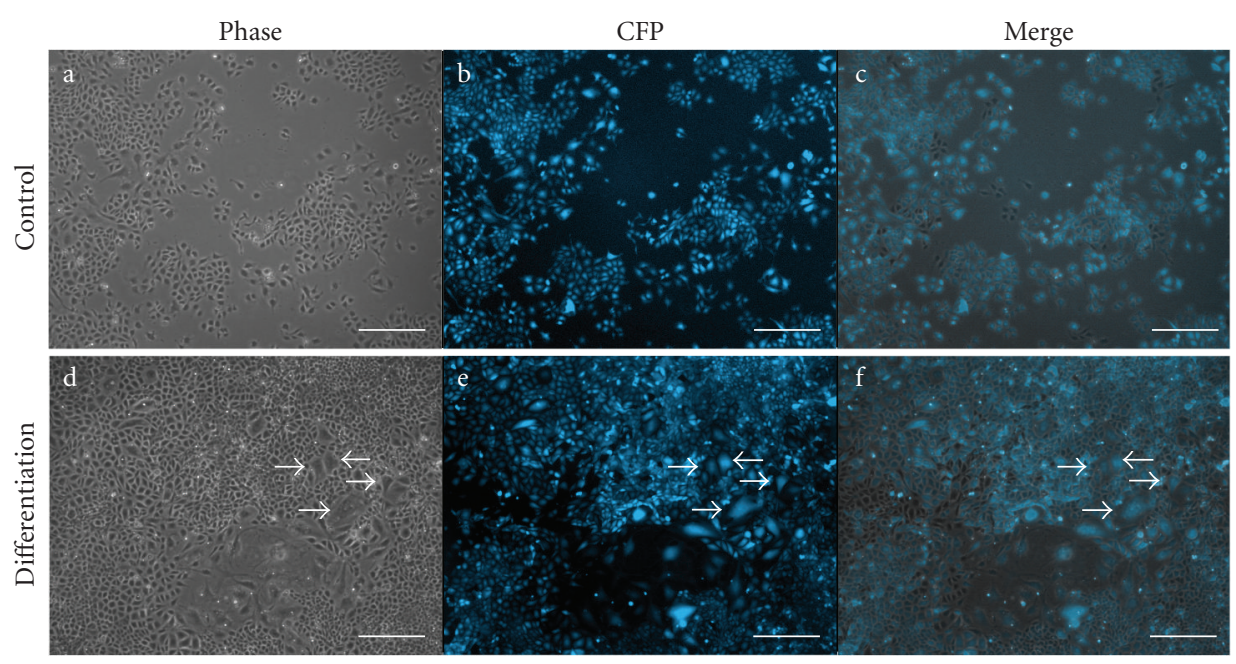

FIGURE 4: Qualitative comparison of CFP-fluorescent B3 cells under control and differentiating culture conditions. Clone B3 cells were cultured under routine (control) (a)-(c) or differentiating (d)-(f); $20 \mathrm{ng} / \mathrm{ml}$ epidermal growth factor, $0.5 \mathrm{ng} / \mathrm{ml}$ transforming growth factor b, $1 \%$ serum) culture conditions for 9 days. Shown are phases (a), (d), fluorescent (b), (e), and merged (c), (f) images. Arrows indicate morphologically differentiated cells. Scale bar is equivalent to $100 \mu \mathrm{m}$.

TABLE 2: Quantitative comparison of the CFP-fluorescent cell fractions of cultures under undifferentiated and differentiated conditions. Flow cytometry quantification of the R2 region of flow histograms (see, e.g., in Figure 5) for three fluorescent cell clones (same as Figure 2). Data are the average \% fluorescent cells at 24 weeks in cultures \pm standard deviation (SD). Cells were analyzed under normal culture conditions (undifferentiated) and under conditions that increase differentiated progeny (differentiation), as described in the text.

\begin{tabular}{ccc}
\hline & \% CFP fluorescence $($ mean $\pm \mathrm{SD} ; n=2)$ condition \\
\hline Strain & Undifferentiated & Differentiated \\
\hline B1 & $94 \pm 0.02$ & $94 \pm 0.88$ \\
B2 & $33 \pm 1.6$ & $28 \pm 0.87$ \\
B3 & $87 \pm 0.32$ & $79 \pm 2.3$ \\
\hline
\end{tabular}

under differentiating conditions (see also Figure 5 for FACS histogram of B3 clone). The B2 and B3 cell clones showed only modest (15\% and 9\%, resp.), albeit statistically significant $(P<.03$ and $P<.02$, resp. $)$, reductions in fluorescent cell fractions (Figure 5; Table 2). Thus, although the three cell strains were derived from three independent clones and displayed differing fluorescent cell fractions (Figure 3), their fluorescence fraction did not vary by more than $15 \%$ when differentiated progeny cells were produced. The estimated fraction of differentiated progeny under these conditions is $\geq 80 \%$ [23], indicating that a majority of differentiated cells retain a high level of fluorescence.

\section{DISCUSSION}

This report is a first study to look at GFP-, CFP-, and YFP-transgenes in side-by-side experiments in the same ASC strain. We evaluated the use of these transgenes for the derivation of stable, long-term fluorescence-expressing rat hepatic ASC clones. We were able to attain transient GFP-expressing cells, but due to either the toxicity and/or methylation associated with GFP, were unable to propagate these clones as stable, long-term GFP-expressing hepatic ASC strains. Given the failure to even establish clones from colonies with extinguished GFP fluorescence, cytotoxicity seems to be the primary problem.

GFP gene transfections gave rise to transient expressing cells for up to 72-96 hours posttransfection. As culture continued, intact, adherent cells positive for GFP-expression began to round up, detach, and lose viability, as indicated by propidium iodide (PI) staining. Eventually, all adherent, GFP-expressing cells rounded up, detached, and became positive for PI staining. These experiments were performed with three different GFP plasmid constructs (pEGFP-N3, pCXEGFP, and pEGFP-N1). However, in no case were stable GFPexpressing cell strains obtainable. These observations, cumulatively, indicate that the GFP protein is toxic to the cells.

Other studies $[15,16]$ have obtained similar results using various GFP expression plasmids. One group in particular [15] examined several variants of GFP plasmids resulting in many of the GFP-expressing cells contracting, rounding up, and dying, which was confirmed by decreasing luciferase activity and increasing CPP32-activity, a cysteine protease that plays a direct role in the proteolytic digestion of cellular proteins responsible for progression to apoptosis.

Our work with GFP confirms that the GFP protein product has toxic side-effects in at least one type of ASC, whereas the CFP and YFP protein, translated from the same plasmid vector construct is well tolerated by these cells. This conclusion can also explain the transfection efficiency and cloning efficiency data from our analyses. Since stable transfection efficiency is an indicator of the success for the transfer and integration of genes into cells, it is likely that due to GFP-related toxicity, both CFP- and YFP-genes transfected 


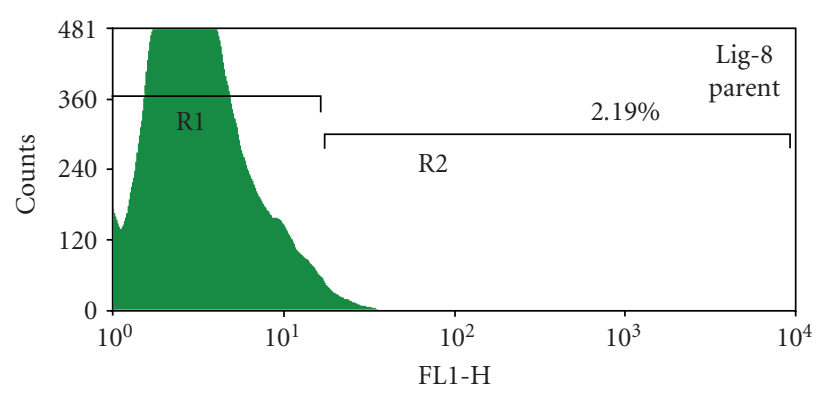

(a)

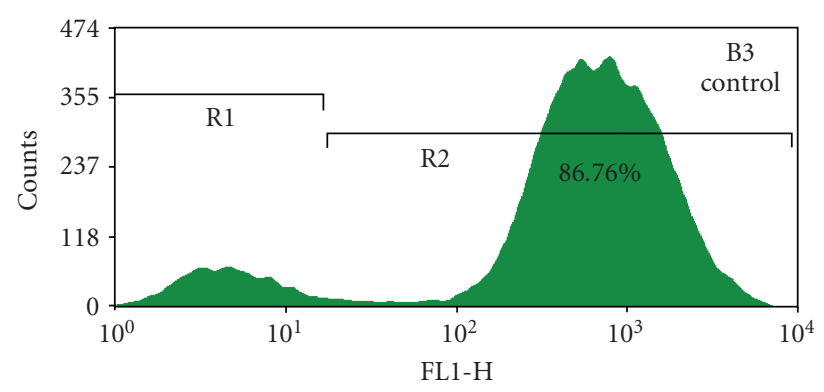

(b)

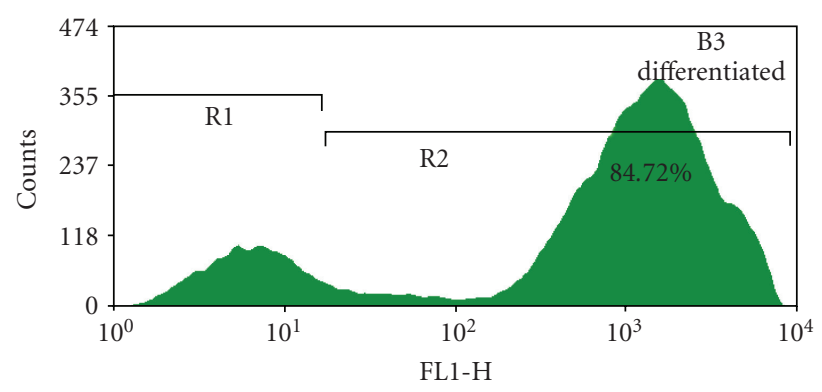

(c)

FIGURE 5: CFP-fluorescence expression of the rat hepatic ASC clone $B 3$ is stable after induction of differentiation. Flow cytometry analysis was performed with cultures of the nontransfected parental rat hepatic ASC strain, Lig-8 (a), and CFP-fluorescent clone B3 hepatic ASC cultures. The B3 cells were evaluated under routine (b) and differentiating (c) culture conditions. Histograms plot the relative numbers of cells as a function of the log-relative CFP fluorescence per cell. The background fluorescence, as defined by nontransfected Lig- 8 cells is depicted as the $\mathrm{R} 1$ region, and positive fluorescence is denoted by the $\mathrm{R} 2$ region. Numbers indicate the percent of cells positive for CFP fluorescence expression.

the hepatic ASCs 50-fold better than the analogous GFP gene. Cloning efficiency data further confirms the difficulties observed with stable, long-term transduction with GFP; since $100 \%$ of CFP- and YFP-clones gave rise to cell strains, whereas none of the GFP-derived colonies gave rise to stable clones. Examined CFP-expressing clones B1, B2, and B3 retained a high level of fluorescent expression at 24 weeks of culture (approximately 140 population doublings), even though one of the clones (B1) initially showed decreasing expression. Altogether, these observations suggest further ev- idence that due to GFP-dependent toxicity, GFP cannot be utilized as a stable fluorescent reporter in these hepatic ASCs.

When the CFP-expressing cell strains (B1, B2, and B3) were placed under differentiation conditions after 120 doublings, either no or only modest reductions in fluorescence cell fractions were observed relative to normal culture conditions. However, although exhibiting stable fluorescence expression under differentiating conditions, B1 and B3 cells displayed a statistically significant increase in the median fluorescence of positive cells. This increase in median fluorescence may be directly related to an increase in the median cell size of the population, since it has been observed that as Lig- 8 cells differentiate, they produce large hepatocyte-like cells [23]. Nonetheless, this stability in fluorescence is important, since it suggests that CFP is expressed independent of the differentiation state of progeny cells. Stable fluorescence expression in our in vitro differentiation studies is a predictor of stable expression in in vivo differentiation, since TGF-b is a ubiquitous differentiation growth factor, especially in the liver [26]. The high stability in fluorescence expression under both normal and differentiating conditions can ideally be used in transplantation studies to evaluate the in vivo repopulation properties of these cells.

The importance of this report is underscored by reports concluding statistically significant amplification of hematopoietic stem cells (HSCs) by forced expression of specific genes. For instance, the gene transfer of the HOXB4 gene into human hematopoietic stem cells was reported to result in an overall approximately 2 -fold increase in total and CD $34^{+}$cells, normalized to a transfer of a control EGFP gene construct [27]. This 2-fold increase and eventual significant overall increase in in vivo repopulation efficiency caused by HOXB4 regulation could be interpreted as the result of increased cell death in EGFP-controls due to fluorescent toxicity and not due to the expansion of HSCs using HOXB4 regulation, as suggested. Similarly, in another report using recombinant HIV transactivating (TAT)-HOXB4 protein [28], TAT-GFP was used as a control for the in vivo expansion and pluripotency of HSCs. It was concluded that TAT-GFP was ineffective in supporting HSC expansion, whereas TATHOXB4 resulted in a net expansion of 20 -fold over control values. Again, this data could result from the toxicitydependent effects of the GFP gene, used as the control for gene transfer.

Availability of stable, long-term marked ASCs has important applications in advancing ASC research. Currently, there are no exclusive ASC markers that allow for easy characterization and validation. One example is the expansion of HSCs in culture, currently a major challenge in the field of HSC research. Although markers have been found that promote enrichment of ASCs from specific tissues $[29,30]$, these are not sufficient for determining their "stemness" in any general sense. Currently, the main method used to establish the "stemness" of an ASC population is transplantation of cells into animals and subsequent determination of whether the cells can regenerate damaged tissues.

In some tissue models, determining repopulation efficiency is simpler than in others. For bone marrow repopulation studies, the output metric is reconstitution of viable 
recipient animals after donor cell transplant. Few ASC studies have this ideal feature of functional reconstitution. Most of these studies depend on in situ cell histology to indicate effective tissue repopulation. Studies of this sort have led to debates regarding issues of ASC plasticity [31]. If a faithful cell marker is not tracked in transplanted ASCs, then uncertainty arises; since it is not clear whether the transplanted cells or host cells are responsible for the observed results. In some tissue models, such as the liver, where the tissue has the capacity for active proliferation, tracking of transplanted cells is even more crucial. Our findings suggest that CFP and YFP are better reporters for the development of stable, longterm fluorescence-expressing ASCs in culture. Their choice for future ASC research may help to resolve current controversial issues, including ASC plasticity in animal repopulation assays.

Notwithstanding the current controversy regarding ASC plasticity and cell fusion [31-33], our findings with GFP call for reevaluation of conclusions based on the transduction of GFP-transgenes into manipulated ASC populations. Additionally, our findings establish important quality control concepts for developing and implementing methods and tools for future ASC therapeutics that employ gene transfer. Our experience highlights the importance of careful in vitrocharacterization of genetically marked cell populations before in vivo transplantation.

\section{ACKNOWLEDGMENTS}

Many thanks to Drs. E. Ozbudak and A. van Oudenaarden for their kind gifts of the pCFP-N3 and pYFP-N3 plasmid constructs, Drs. C. Semino and S. Zhang for their assistance with fluorescent microscopy, G. Paradis and the MIT Flow Cytometry Core Facility staff for their assistance and expertise in flow cytometry analyses, and A. Nichols, S. Ram-Mohan, K. Panchalingam, Drs. J.F. Paré, J. Lansita, and G. Crane for review of this manuscript. This research was supported by NSF Engineering Research Center Grant no. 9843342. R. R. Taghizadeh was supported by NIH/NIGMS Interdepartmental Biotechnology Training Program Grant no. 2 T32 GM08334, and National Institutes of Health Director's Pioneer Award no. 5DP10D000805-02.

\section{REFERENCES}

[1] C. W. Cody, D. C. Prasher, W. M. Westler, F. G. Prendergast, and W. W. Ward, "Chemical structure of the hexapeptide chromophore of the Aequorea green-fluorescent protein," Biochemistry, vol. 32, no. 5, pp. 1212-1218, 1993.

[2] M. Chalfie, Y. Tu, G. Euskirchen, W. W. Ward, and D. C. Prasher, "Green fluorescent protein as a marker for gene expression," Science, vol. 263, no. 5148, pp. 802-805, 1994.

[3] Y. Li and M. S. Horwitz, "Use of green fluorescent protein in studies of apoptosis of transfected cells," BioTechniques, vol. 23, no. 6, pp. 1026-1029, 1997.

[4] Y. Yoon, K. Pitts, and M. McNiven, "Studying cytoskeletal dynamics in living cells using green fluorescent protein," Molecular Biotechnology, vol. 21, no. 3, pp. 241-250, 2002.

[5] Y.-X. Zeng, K. Somasundaram, N. S. Prabhu, R. Krishnadasan, and W. S. El-Deiry, "Detection and analysis of living, growth- inhibited mammalian cells following transfection," BioTechniques, vol. 23, no. 1, pp. 88-94, 1997.

[6] S. Wang and T. Hazelrigg, "Implications for $b c d$ mRNA localization from spatial distribution of exu protein in Drosophila oogenesis," Nature, vol. 369, no. 6479, pp. 400-403, 1994.

[7] A. Amsterdam, S. Lin, and N. Hopkins, "The Aequorea victoria green fluorescent protein can be used as a reporter in live zebrafish embryos," Developmental Biology, vol. 171, no. 1, pp. 123-129, 1995.

[8] K. G. Peters, P. S. Rao, B. S. Bell, and L. A. Kindman, "Green fluorescent fusion proteins: powerful tools for monitoring protein expression in live zebrafish embryos," Developmental Biology, vol. 171, no. 1, pp. 252-257, 1995.

[9] T. Takada, K. Iida, T. Awaji, et al., "Selective production of transgenic mice using green fluorescent protein as a marker," Nature Biotechnology, vol. 15, no. 5, pp. 458-461, 1997.

[10] M. Ikawa, K. Kominami, Y. Yoshimura, K. Tanaka, Y. Nishimune, and M. Okabe, "Green fluorescent protein as a marker in transgenic mice," Development, Growth \& Differentiation, vol. 37, no. 4, pp. 455-459, 1995.

[11] M. Ikawa, K. Kominami, Y. Yoshimura, K. Tanaka, Y. Nishimune, and M. Okabe, "A rapid and non-invasive selection of transgenic embryos before implantation using green fluorescent protein (GFP)," FEBS Letters, vol. 375, no. 1-2, pp. 125-128, 1995.

[12] M. Okabe, M. Ikawa, K. Kominami, T. Nakanishi, and Y. Nishimune, "'Green mice' as a source of ubiquitous green cells," FEBS Letters, vol. 407, no. 3, pp. 313-319, 1997.

[13] Y. Hakamata, K. Tahara, H. Uchida, et al., "Green fluorescent protein-transgenic rat: a tool for organ transplantation research," Biochemical and Biophysical Research Communications, vol. 286, no. 4, pp. 779-785, 2001.

[14] W. C. Kisseberth, N. T. Brettingen, J. K. Lohse, and E. P. Sandgren, "Ubiquitous expression of marker transgenes in mice and rats," Developmental Biology, vol. 214, no. 1, pp. 128-138, 1999.

[15] H.-S. Liu, M.-S. Jan, C.-K. Chou, P.-H. Chen, and N.-J. Ke, "Is green fluorescent protein toxic to the living cells?" Biochemical and Biophysical Research Communications, vol. 260, no. 3, pp. 712-717, 1999.

[16] L. Lybarger, D. Dempsey, K. J. Franek, and R. Chervenak, "Rapid generation and flow cytometric analysis of stable GFPexpressing cells," Cytometry, vol. 25, no. 3, pp. 211-220, 1996.

[17] S. J. Kromenaker and F. Srienc, "Stability of producer hybridoma cell lines after cell sorting: a case study," Biotechnology Progress, vol. 10, no. 3, pp. 299-307, 1994.

[18] M. Zeyda, N. Borth, R. Kunert, and H. Katinger, "Optimization of sorting conditions for the selection of stable, high-producing mammalian cell lines," Biotechnology Progress, vol. 15, no. 5, pp. 953-957, 1999.

[19] K. Hong, J. L. Sherley, and D. A. Lauffenburger, "Methylation of episomal plasmids as a barrier to transient gene expression via a synthetic delivery vector," Biomolecular Engineering, vol. 18, no. 4, pp. 185-192, 2001.

[20] R. Y. Tsien, “The green fluorescent protein,” Annual Review of Biochemistry, vol. 67, pp. 509-544, 1998.

[21] R. Heim, D. C. Prasher, and R. Y. Tsien, "Wavelength mutations and posttranslational autoxidation of green fluorescent protein," Proceedings of the National Academy of Sciences of the United States of America, vol. 91, no. 26, pp. 12501-12504, 1994. 
[22] H.-S. Lee, G. G. Crane, J. R. Merok, et al., "Clonal expansion of adult rat hepatic stem cell lines by suppression of asymmetric cell kinetics (SACK)," Biotechnology and Bioengineering, vol. 83, no. 7, pp. 760-771, 2003.

[23] G. G. Crane, R. R. Taghizadeh, and J. L. Sherley,in preparation.

[24] J. L. Sherley, "Asymmetric cell kinetics genes: the key to expansion of adult stem cells in culture," Stem Cells, vol. 20, no. 6, pp. 561-572, 2002.

[25] C. E. Semino, J. R. Merok, G. G. Crane, G. Panagiotakos, and S. Zhang, "Functional differentiation of hepatocyte-like spheroid structures from putative liver progenitor cells in three-dimensional peptide scaffolds," Differentiation, vol. 71, no. 4-5, pp. 262-270, 2003.

[26] A. Sánchez, R. Pagan, A. M. Álvarez, et al., “Transforming growth factor- $\beta$ (TGF- $\beta$ ) and EGF promote cord-like structures that indicate terminal differentiation of fetal hepatocytes in primary culture," Experimental Cell Research, vol. 242, no. 1, pp. 27-37, 1998.

[27] S. Amsellem, F. Pflumio, D. Bardinet, et al., "Ex vivo expansion of human hematopoietic stem cells by direct delivery of the HOXB4 homeoprotein," Nature Medicine, vol. 9, no. 11, pp. 1423-1427, 2003.

[28] J. Krosl, P. Austin, N. Beslu, E. Kroon, R. K. Humphries, and G. Sauvageau, "In vitro expansion of hematopoietic stem cells by recombinant TAT-HOXB4 protein,” Nature Medicine, vol. 9, no. 11, pp. 1428-1432, 2003.

[29] N. Uchida and I. L. Weissman, "Searching for hematopoietic stem cells: evidence that Thy- $1.1^{\mathrm{Io}} \mathrm{Lin}^{-} \mathrm{Sca}-1^{+}$cells are the only stem cells in C57BL/Ka-Thy-1.1 bone marrow," Journal of Experimental Medicine, vol. 175, no. 1, pp. 175-184, 1992.

[30] G. J. Spangrude, S. Heimfeld, and I. L. Weissman, "Purification and characterization of mouse hematopoietic stem cells," Science, vol. 241, no. 4861, pp. 58-62, 1988.

[31] A. J. Wagers, R. I. Sherwood, J. L. Christensen, and I. L. Weissman, "Little evidence for developmental plasticity of adult hematopoietic stem cells," Science, vol. 297, no. 5590, pp. 2256-2259, 2002.

[32] A. J. Wagers and I. L. Weissman, "Plasticity of adult stem cells," Cell, vol. 116, no. 5, pp. 639-648, 2004.

[33] F. D. Camargo, S. M. Chambers, and M. A. Goodell, "Stem cell plasticity: from transdifferentiation to macrophage fusion," Cell Proliferation, vol. 37, no. 1, pp. 55-65, 2004. 


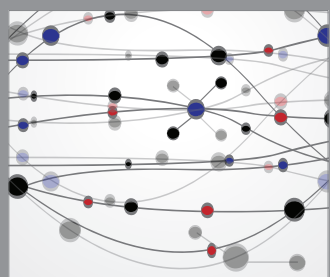

The Scientific World Journal
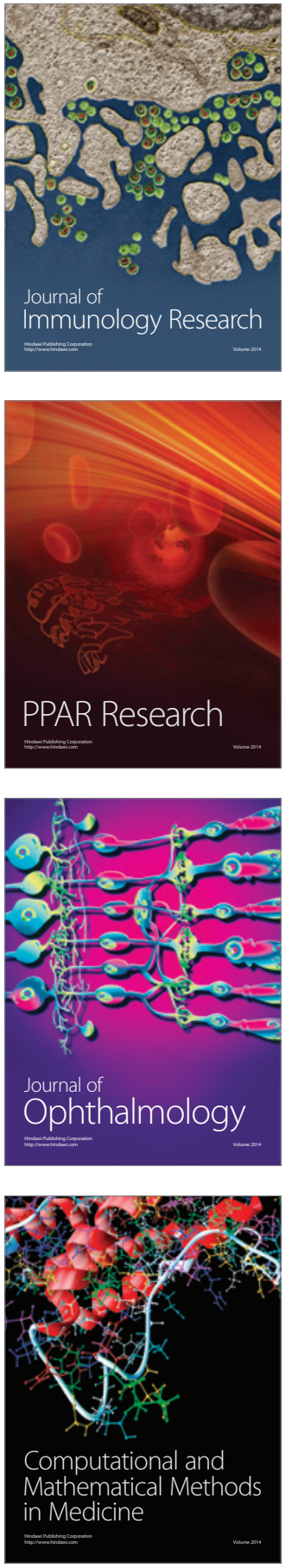

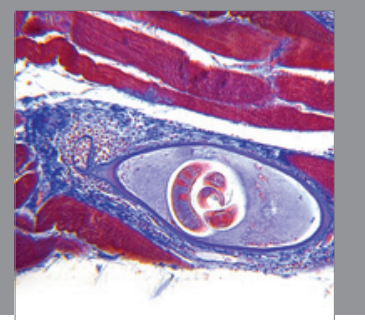

Gastroenterology

Research and Practice
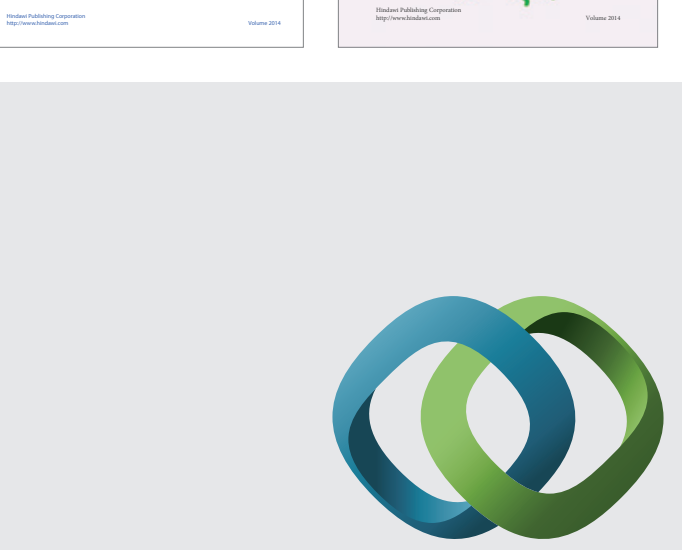

\section{Hindawi}

Submit your manuscripts at

http://www.hindawi.com
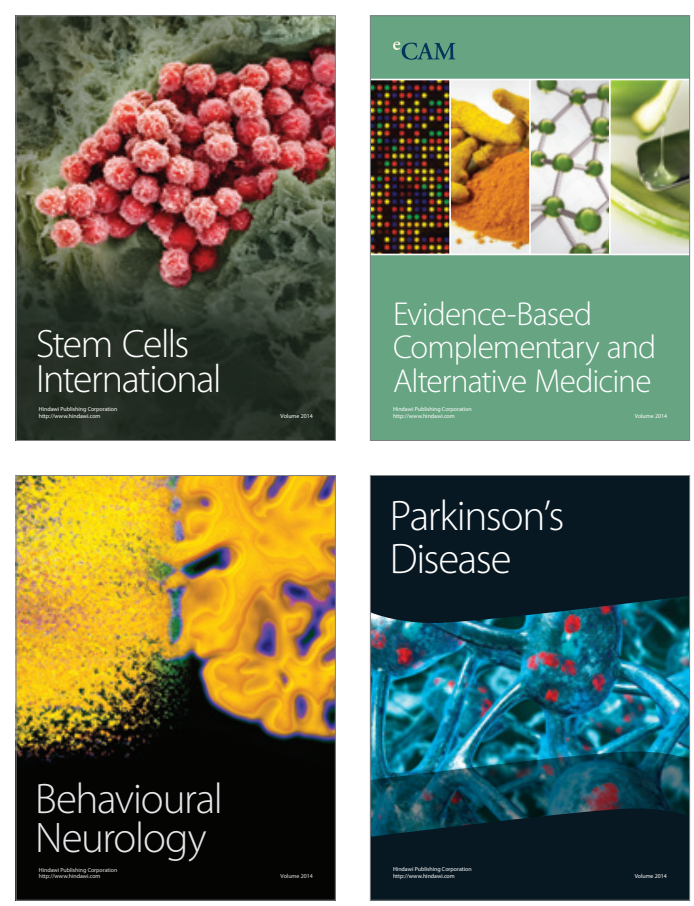

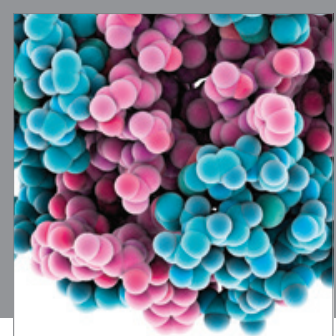

Journal of
Diabetes Research

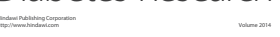

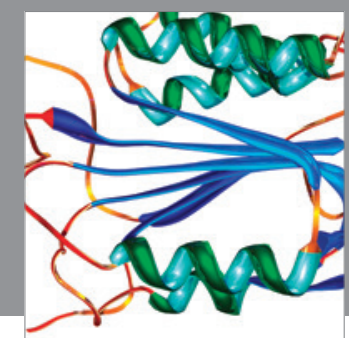

Disease Markers
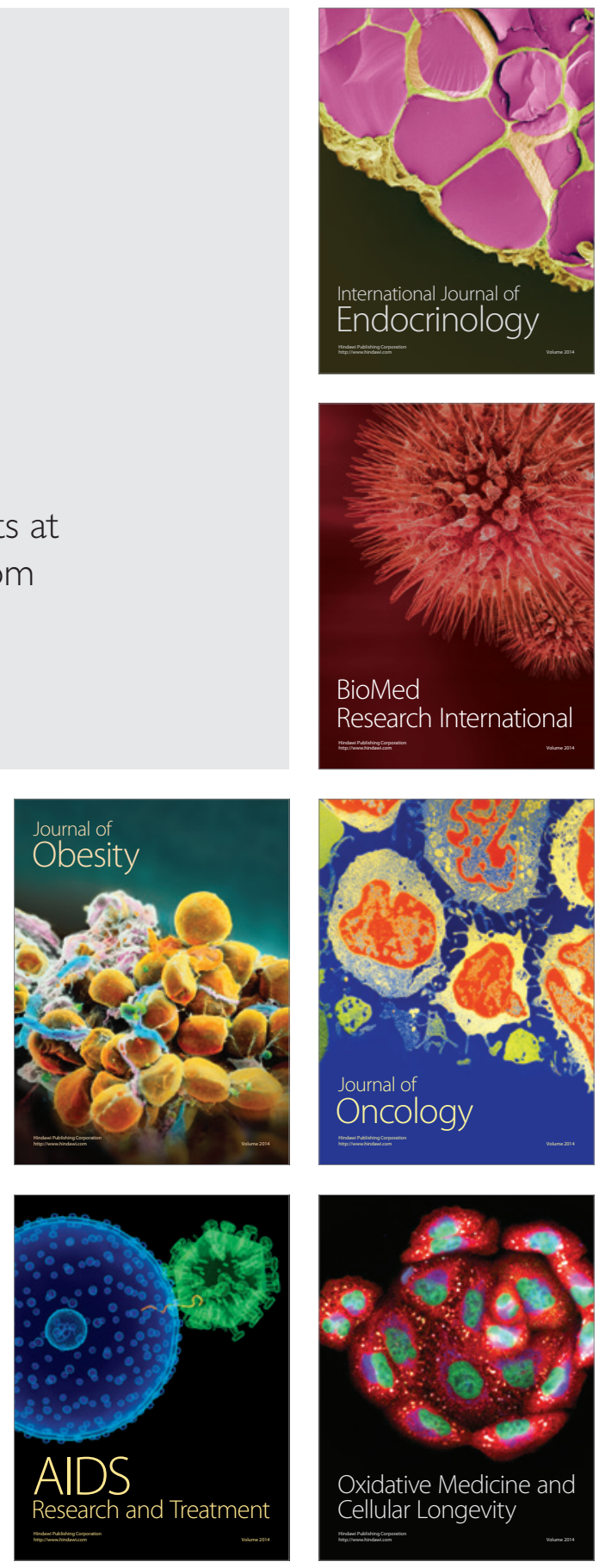